\title{
A mathematical framework for group analysis of von Willebrand factor multimeric composition following luminography
}

A.A. Lopes ${ }^{1}$,

R.P.S. Soares ${ }^{2}$ and N.Y. Maeda ${ }^{2}$
1Departamento de Cardiologia Pediátrica e Cardiopatias Congênitas, Instituto do Coração, Universidade de São Paulo, and 'Fundação Pró-Sangue, São Paulo, SP, Brasil

\section{Correspondence}

A.A. Lopes

Departamento de Cardiologia

Pediátrica e Cardiopatias Congênitas

Instituto do Coração, USP

Av. Dr. Eneas C. Aguiar, 44

05403-000 São Paulo, SP

Brasil

Fax: +55-11-3069-5347

E-mail: aablopes@usp.br

Research supported by FAPESP

(No. 00/02845-8).

Received April 23, 2002

Accepted August 6, 2002

\begin{abstract}
The objective of the present study was to establish a method for quantitative analysis of von Willebrand factor (vWF) multimeric composition using a mathematical framework based on curve fitting. Plasma vWF multimers from 15 healthy subjects and 13 patients with advanced pulmonary vascular disease were analyzed by Western immunoblotting followed by luminography. Quantitative analysis of luminographs was carried out by calculating the relative densities of low, intermediate and high molecular weight fractions using laser densitometry. For each densitometric peak (representing a given fraction of vWF multimers) a mean area value was obtained using data from all group subjects (patients and normal individuals) and plotted against the distance between the peak and IgM (950 kDa). Curves were constructed for each group using nonlinear fitting. Results indicated that highly accurate curves could be obtained for healthy controls and patients, with respective coefficients of determination $\left(\mathrm{r}^{2}\right)$ of 0.9898 and 0.9778 . Differences were observed between patients and normal subjects regarding curve shape, coefficients and the region of highest protein concentration. We conclude that the method provides accurate quantitative information on the composition of vWF multimers and may be useful for comparisons between groups and possibly treatments.
\end{abstract}

von Willebrand factor (vWF) is a large multimeric glycoprotein synthesized exclusively by endothelial cells and megakaryocytes (1). Endothelial vWF is secreted into the circulation as high molecular weight multimers and rapidly converted into smaller polymers by the action of proteases, mainly from granulocytes $(2,3)$. As a result, a very typical multimeric pattern of vWF may be observed when plasma is subjected to elec-
Key words

- von Willebrand factor

- Luminography

- Densitometry

- Curve fitting

- Pulmonary hypertension trophoresis in agarose gels, with bands of 500 up to $20,000 \mathrm{kDa}(4,5)$.

The multimeric pattern of plasma $\mathrm{vWF}$ may be changed in acquired and inherited disorders, as is the case for von Willebrand's disease (6). In some instances, unusually large multimers are present in plasma $(7,8)$. In other conditions, high molecular weight multimers are lacking and this may be associated with increased concentrations of low 
molecular weight forms (9-11). Using highresolution gels, changes may also be observed in the structure of individual multimers as reported in variants of von Willebrand's disease (12).

In most instances, qualitative analysis of vWF multimeric composition provides adequate information for characterization of abnormalities and correlation with the clinical status. However, quantitative analysis may be useful for the detection of subtle abnormalities and changes following therapeutical interventions. Quantitative assessment of vWF multimeric composition has been performed on an individual basis by densitometric analysis of Western blots $(13,14)$. In the present study, we propose a method for quantitative estimation of the relative distribution of $\mathrm{vWF}$ multimers in immunoblots based on adjustment of mathematical models following densitometry. As an example of the potential usefulness of the method for group analysis, we studied plasma vWF multimers in a population of patients with advanced pulmonary vascular disease for whom abnormalities in vWF structure have been reported that resemble type II von Willebrand's disease (11). The study protocol was approved by the Scientific Committee of the Heart Institute, São Paulo, Brazil.

Blood samples were drawn after written informed consent was obtained from 15 healthy subjects and 13 patients with advanced pulmonary vascular disease (ages 4 to 50 years, mean pulmonary arterial pressure of 49 to $98 \mathrm{mmHg}$ ) who were admitted to the Heart Institute, São Paulo, SP, Brazil. Peripheral venous blood was collected in $1: 10$ volumes of $3.8 \%$ sodium citrate in the presence of protease inhibitors: $5 \mathrm{mM}$ edetic acid (EDTA), 6 mM N-ethylmaleimide, 1 $\mathrm{mM}$ phenylmethylsulfonyl fluoride, $20 \mathrm{U} /$ $\mathrm{ml}$ aprotinin, and $0.25 \mathrm{mM}$ leupeptin. Plasma was separated by centrifugation at $3,000 \mathrm{~g}$ for $20 \mathrm{~min}$, frozen at $-80^{\circ} \mathrm{C}$ and thawed only once for use.

Plasma $(1 \mu \mathrm{l})$ was diluted 1:20 in $10 \mathrm{mM}$
Tris, 2\% SDS, $1 \mathrm{mM}$ EDTA, $80 \mathrm{mM}$ urea, $0.02 \%$ bromophenol blue, $\mathrm{pH} 8.0$, incubated at $56^{\circ} \mathrm{C}$ for $1 \mathrm{~h}$ and electrophoresed on SDS$1 \%$ low gelling temperature agarose. The running buffer was: $40 \mathrm{mM}$ Tris, $20 \mathrm{mM}$ sodium acetate, 2 mM EDTA, 0.1\% SDS, pH 7.4.

After the run, protein was transferred to a nitrocellulose membrane at a constant current of $200 \mathrm{~mA}$ for $14 \mathrm{~h}$, using a minielectrotransfer unit (Hoefer TE 22 transphor unit, Amersham Pharmacia Biotech, Little Chalfont, Buckinghamshire, England) to keep a constant temperature of $15^{\circ} \mathrm{C}$. Transfer buffer was: $25 \mathrm{mM}$ Tris, $192 \mathrm{mM}$ glycine, $0.1 \%$ SDS, and $20 \%(\mathrm{v} / \mathrm{v})$ methanol, $\mathrm{pH} 8.3$. After blocking the membrane with 5\% BSA, immobilized protein was reacted for $6 \mathrm{~h}$ with rabbit anti-human vWF polyclonal antibody (1:300 dilution) (Dako Corporation, Carpinteria, CA, USA).

All steps for protein visualization followed the instructions of the chemiluminescence kit manufacturer (ECL Western blot detection kit, Amersham Pharmacia Biotech). Peroxidase-conjugated secondary antibody was used at 1:2000 dilution. Membranes were then reacted with a high sensitivity (luminol) detection system for $1 \mathrm{~min}$ and exposed to X-ray films for 10 to $30 \mathrm{~s}$.

vWF multimers were subjected to densitometric analysis (Ultroscan XL laser densitometer, LKB, Bromma, Sweden) using IgM (molecular mass $950 \mathrm{kDa}$ ) as a reference. Scanning was started at the IgM position which corresponded to low molecular weight vWF multimers and run through the intermediate and high molecular weight forms. In most preparations, 13 to 14 peaks were accurately analyzed. The densitometric area of individual peaks was computed and normalized for the area of the first major band migrating just above IgM. This procedure was necessary to eliminate the influence of differences between lanes in total amount of vWF protein.

For each normalized peak, a mean area 
value was obtained using data from all group subjects (patients or normal subjects) and plotted as a function of the distance between the peak and IgM. Thus, mean curves were constructed for specific groups, representing the relative amounts of vWF protein in different multimeric fractions. Curve fitting was performed using the SAS/STAT ${ }^{\circledR}$ pack (SAS Institute Inc., Cary, NC, USA). The best fit was obtained by analyzing up to 30 different nonlinear models using a stepwise procedure. The goodness of fit was determined by calculating the coefficient of determination $\left(\mathrm{r}^{2}\right)$.

Details of Western immunoblotting and densitometric scanning are shown in Figure 1 . Figure 2 shows the plots of mean densitometric areas against the distance between the respective peaks and the IgM band. Although the same model was adequately adjusted to patients and normal subjects, the morphology of the curves was different, as indicated by the respective equation coefficients. In normal subjects, there was a relatively high concentration of vWF multimers at $36.4 \mathrm{~mm}$ from IgM. In contrast, patients had a flattened curve, with highest multimer concentration at $27.9 \mathrm{~mm}$ from the IgM position. The right terminal of the curve, representing the relative area of high molecular weight multimers, was markedly lowered in patients compared to normal subjects.

Peroxidase staining methods have been developed for analysis of the multimeric structure of vWF (15-17). Further improvement of sensitivity has been reported using luminography for protein visualization after electrophoresis $(18,19)$. Although densitometric analysis of immunoblots has proved useful for determining the distribution of vWF multimers in individual patients, as reported in different forms of von Willebrand's disease (14), group evaluation may be necessary in some instances and requires a different approach. This may be the case for acquired disorders with more subtle abnormalities of vWF structure or evaluation of response to treatment (20).

In individual analysis of vWF structure, results of densitometric tracings may be expressed, for example, as the relative area of larger vWF multimers (13). In the present study, we used a mathematical approach for group analysis of vWF multimeric composition and observed that nonlinear models fit plots of mean densitometric area values very nicely as a function of distance. As a result, differences between groups may be estimated not only by calculating the percentage of larger multimers, but also using a number of other parameters such as the best curve fit

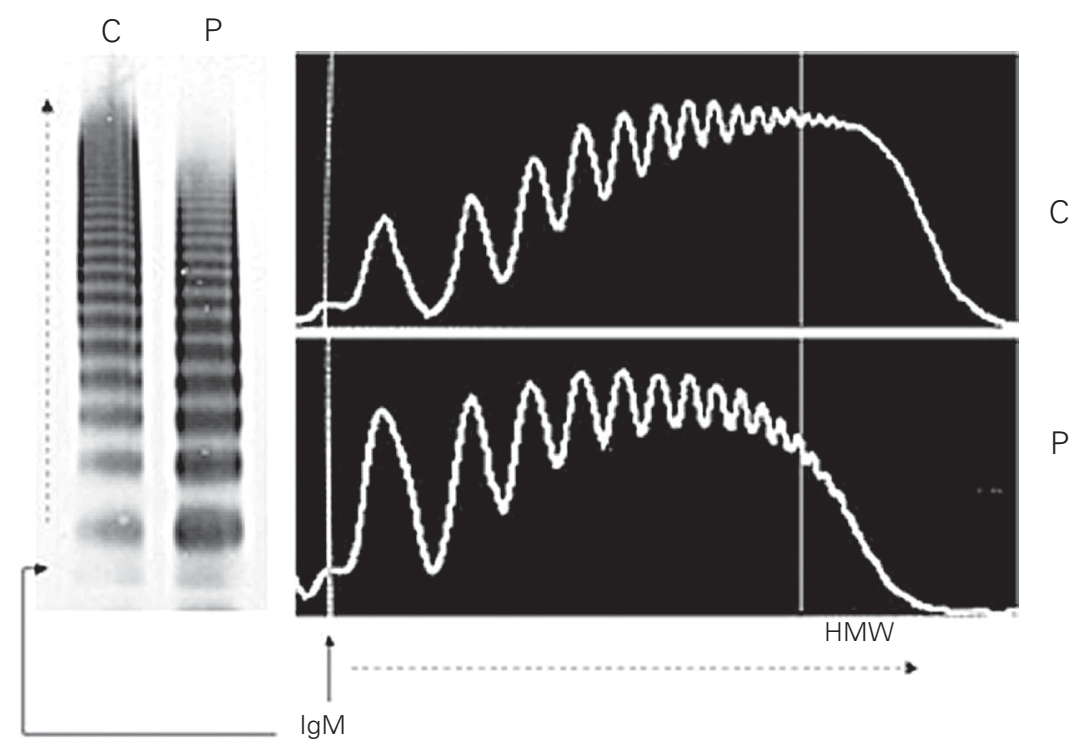

Figure 1. Representative densitometric tracing of vWF multimers in Western blots. Scanning was started at the IgM position (arrows, low molecular weight fractions) and run as indicated by the dashed lines in the direction of high molecular weight multimers (HMW). C and $\mathrm{P}$ are control plasma and patient plasma, respectively.

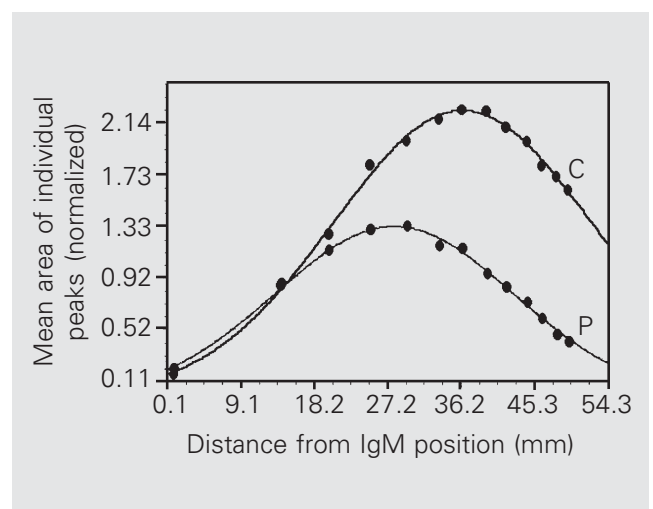

Figure 2. Mean curves for relative amounts of VWF protein in different multimeric fractions. Each point represents the mean densitometric area of a given peak. A Gaussian model [y $=$ $\left.a^{*} \exp \left(\left(-(x-b)^{\wedge} 2\right) /\left(2{ }^{*} c^{\wedge} 2\right)\right)\right]$ was adequately adjusted to controls $(C$, $\left.r^{2}=0.9898\right)$ and patients $\left(P, r^{2}=\right.$ $0.9778)$. The respective equation coefficients were: $a=2.2321, b$ $=36.3822, \mathrm{c}=15.8908$ for control group $(\mathrm{N}=15)$, and $\mathrm{a}=$ $1.3378, b=27.8795, c=14.6598$ for patient group ( $N=13$ ). 
for a given group, curve coefficients and the region of highest concentration of VWF protein. Comparisons between groups may be performed at any region of the multimer distribution. Furthermore, the relative density of high molecular weight multimers which are sometimes poorly individualized in densitometric scans may be estimated for the whole group using the curve equation.

Previous studies on the multimeric structure of plasma vWF in pulmonary hypertension, including data from our laboratory, showed the disappearance of the larger multimers, associated with the increased density of the smaller ones $(11,20)$. In this study, we were able to identify the exact region of highest vWF protein concentration in the sequence of multimers. We therefore speculate that the present method could be helpful, for example, to quantify the improvement of multimeric abnormalities following therapeutical interventions in the same patients (20).

Methodological details were taken into consideration in this study in order to improve reliability, reproducibility and comparability of the results. First, protein transfer to nitrocellulose membranes was carried out over a period of $14 \mathrm{~h}$ to permit the complete transfer of all vWF multimers, in particular the larger ones. Second, saturation of the luminographs was prevented by limiting the exposure time to a maximum of $30 \mathrm{~s}$. Third, the area of the densitometric peaks was expressed as a percentage of the first major band migrating above IgM. This within-lane normalization procedure allowed us to compute data from different lanes regardless of the exact amount of $\mathrm{vWF}$ protein applied per well. Last, only high quality luminographs were included in the study, making it possible to analyze at least 13 multimeric peaks per lane. This resulted in adequate curve fit with high coefficients of determination $\left(\mathrm{r}^{2}\right)$ when mean densitometric area values were analyzed.

In view of the present observations, we conclude that curve fitting with an adequate number of points representing mean area values is useful for group analysis of vWF multimeric structure. Although obvious abnormalities of multimer distribution were presented here as an example of the potential usefulness of the method, we believe that subtle between-group differences can be accurately demonstrated as well. Additional studies are required, enrolling patients with other disorders or subjected to therapeutical interventions to confirm the usefulness of this method in clinical practice and research.

\section{References}

1. Sadler JE (1991). von Willebrand factor. Journal of Biological Chemistry, 266: 22777-22780.

2. Tsai H-M, Nagel RL, Hatcher VB \& Sussman II (1989). Multimeric composition of endothelial cell-derived von Willebrand factor. Blood, 73: 2074-2076.

3. Tsai H-M, Nagel RL, Hatcher VB \& Sussman II (1989). Endothelial cellderived high molecular weight von Willebrand factor is converted into the plasma multimer pattern by granulocyte proteases. Biochemical and Biophysical Research Communications, 158: 980-985.

4. Ruggeri ZM \& Zimmerman TS (1981). The complex multimeric composition of factor VIII/von Willebrand factor. Blood, 57:
1140-1143.

5. Bukh A, Ingerslev J, Stenbjerg S \& Hundahl Møller NP (1986). The multimeric structure of plasma FVIII:RAg studied by electroelution and immunoperoxidase detection. Thrombosis Research, 43: 579584.

6. Sadler J (1994). A revised classification of von Willebrand disease. Thrombosis and Haemostasis, 71: 520-525.

7. Mannucci PM, Lombardi R, Lattuada A, Perticucci E, Valsecchi R \& Remuzzi G (1987). Supranormal von Willebrand factor multimers in scleroderma. Blood, 73: 1586-1591.

8. Moake JL (1997). von Willebrand factor in the pathophysiology of thrombotic throm- bocytopenic purpura. Annals of Clinical and Laboratory Science, 11: 362-364.

9. Rinder MR, Richard RE \& Rinder HM (1997). Acquired von Willebrand's disease: a concise review. American Journal of Hematology, 54: 139-145.

10. Pareti Fl, Lattuada A, Bressi C, Zanobini M, Sala A, Steffan A \& Ruggeri ZM (2000). Proteolysis of von Willebrand factor and shear stress-induced platelet aggregation in patients with aortic valve stenosis. Circulation, 102: 1290-1295.

11. Lopes AA, Maeda NY \& Bydlowski SP (1998). Abnormalities in circulating von Willebrand factor and survival in pulmonary hypertension. American Journal of Medicine, 105: 21-26. 
12. Ciavarella G, Ciavarella N, Antoncecchi S, De Mattia D, Ranieri P, Dent J, Zimmerman TS \& Ruggeri ZM (1985). High-resolution analysis of von Willebrand factor multimeric composition defines a new variant of type I von Willebrand disease with aberrant structure but presence of all size multimers (type IC). Blood, 66: 1423-1429.

13. Budde U, Scharf RE, Franke P, HartmannBudde K, Dent J \& Ruggeri ZM (1993). Elevated platelet count as a cause of abnormal von Willebrand factor multimer distribution in plasma. Blood, 82: 17491757.

14. Studt JD, Budde U, Schneppenheim R, Eisert R, von Depka Prondzinski M, Ganser A \& Barthels M (2001). Quantification and facilitated comparison of von Willebrand factor multimer patterns by den- sitometry. American Journal of Clinical Pathology, 116: 567-574.

15. Aihara M, Sawada $Y$, Ueno K, Morimoto S, Yoshida Y, de Serres M, Cooper HA \& Wagner RH (1986). Visualization of von Willebrand factor multimers by immunoenzymatic stain using avidin-biotin peroxidase complex. Thrombosis and Haemostasis, 55: 263-267.

16. Tomita $Y$, Harrison J \& Abildgaard CF (1989). von Willebrand factor multimer analysis using a sensitive peroxidase staining method. Thrombosis and Haemostasis, 62: 781-783.

17. Ledford MR, Kent JW \& Civantos F (1990). A comparative study of three methods for the visualization of von Willebrand factor (vWF) multimers. Thrombosis and Haemostasis, 64: 569-575.

18. Budde $U$, Schneppenheim R, Plendl $H$,
Dent J, Ruggeri ZM \& Zimmerman TS (1990). Luminographic detection of von Willebrand factor multimers in agarose gels and on nitrocellulose membranes. Thrombosis and Haemostasis, 63: 312315.

19. Krizek DR \& Rick ME (2000). A rapid method to visualize von Willebrand factor multimers by using agarose gel electrophoresis, immunolocalization and luminographic detection. Thrombosis Research, 97: 457-462.

20. Veyradier A, Nishikubo $T$, Humbert $M$, Wolf M, Sitbon O, Simonneau G, Girma J-P \& Meyer D (2000). Improvement of von Willebrand factor proteolysis after prostacyclin infusion in severe pulmonary arterial hypertension. Circulation, 102: 2460-2462. 